\title{
Relationships and Health among Emerging Adults with and without Type 1 Diabetes
}

\author{
Vicki S. Helgeson and Dianne K. Palladino \\ Carnegie Mellon University
}

Kerry A. Reynolds

Rand Association

Dorothy J. Becker and Oscar Escobar

Children's Hospital of Pittsburgh

\section{Linda Siminerio \\ University of Pittsburgh Medical Center}

\begin{abstract}
Objective-The study's goal was to examine the impact of parent and peer relationships on health behaviors and psychological well-being of those with and without type 1 diabetes over the transition to emerging adulthood. Emerging adulthood is an understudied developmental period and a high risk period—especially for those with type 1 diabetes.
\end{abstract}

Methods-Youth with $(\mathrm{n}=117)$ and without type 1 diabetes $(\mathrm{n}=122)$ completed questionnaires during their senior year of high school and one year later. Measures included supportive and problematic aspects of parent and peer relationships, health behaviors, psychological well-being, and, for those with diabetes, self-care behavior and glycemic control.

Results-Prospective multiple and logistic regression analysis revealed that friend conflict was a more potent predictor than friend support of changes in health behaviors and psychological wellbeing. Parent support was associated with positive changes in psychological well-being and decreases in smoking, whereas parent control was related to increases in smoking and depressive symptoms. There was some evidence of cross-domain buffering such that supportive relationships in one domain buffered adverse effects of problematic relationships in the other domain on health outcomes.

Conclusions-This longitudinal study showed that parent relationships remain an important influence on and peer relationships continue to influence the health behaviors and psychological well-being of emerging adults with and without type 1 diabetes. Parent relationships also have the potential to buffer the adverse effects of difficulties with peers.

\section{Keywords}

social support; health behavior; psychological well-being; glycemic control; diabetes

Correspondence concerning this article should be addressed to Vicki S. Helgeson, Department of Psychology, Carnegie Mellon University, Pittsburgh, PA 15213; vh2e@andrew.cmu.edu. 
Emerging adulthood, the developmental period between the ages of 18 and 25 (Arnett, 2000 ), is a period characterized by exploration in a variety of life domains. From a vocational perspective, many youth graduate from high school and further their education or enter the labor force. From a relational perspective, young adults continue to separate from their families of origin and form even stronger attachments to peers. These choices lead to changes in one's social environment, and an evolving social environment may affect emerging adults' health.

The social environment during adolescence consists of relationships with both family and peers (Brown, Boeving, LaRosa, \& Carpenter, 2006). Over the course of adolescence, youth become increasingly independent from parents and increasingly immersed in relationships with peers (Holmbeck, Friedman, Abad, \& Jandasek, 2006). These two social systems continue to evolve during emerging adulthood. Parents are no longer a constant in the social environment of emerging adults, yet there is evidence that parents have a continuing influence on their lives. Studies of college students have shown that parent encouragement of autonomy (rather parent controlling behavior) is associated with less risk behavior (Haemmerlie, Steen, \& Benedicto, 1994; Sessa, 2005) and greater well-being (Kins, Beyers, Soenens, \& Vansteenkiste, 2009). Although peer influence is said to peak during early/ middle adolescence (Steinberg \& Monahan, 2007), emerging adults spend the majority of their time with others their own age. Studies of college students have shown that peer norms regarding alcohol and peer influence are linked to risk behaviors, especially alcohol problems (Allison, 2003; Borsari \& Carey, 2001; Wood, Read, Mitchell, \& Brand, 2004). Just as the two systems interact to influence adolescent health (Holmbeck, 2002), these changing family and peer systems are likely to have implications for the health of emerging adults.

Emerging adulthood is a time in which health and well-being outcomes are of great importance. This age group has the highest rate of alcohol and drug usage, unprotected sex, and driving while intoxicated (Arnett, 2007; Frech, 2009). Mental health issues are prominent. Depressive symptoms increase during adolescence, reaching their highest rates during emerging adulthood, and then decline over the rest of the life span (Arnett, 2004).

Although eating disorders and eating disturbances typically appear in early to middle adolescence, the age of onset for bulimia is late adolescence and early adulthood (American Psychiatric Association, 2000). Behaviors that appear during emerging adulthood may set in motion a pattern that persists through adulthood and threatens health. Thus, it is important to examine whether family and peer relationships promote or undermine these behaviors.

The changing social environment associated with emerging adulthood may have even greater health implications for youth who are managing a chronic disease, such as type 1 diabetes. Indeed, emerging adulthood has been identified as a potential risk period in terms of health behavior and physical health among those with type 1 diabetes (Peters \& Laffel, 2011). Unlike the health regimens for many other chronic diseases, those for type 1 diabetes involve significant behavioral commitment throughout each day. Management of type 1 diabetes involves testing blood glucose, administering and adjusting insulin doses, maintaining a healthy diet, and incorporating physical activity on a daily basis. This ubiquitous influence of diabetes management results in an enormous potential for environmental factors, 
including personal relationships, to play a role in proper care of diabetes as well as overall well-being.

There are few studies of the social environment of emerging adults with type 1 diabetes. One study of 16 to 26 -year-olds with type 1 diabetes showed that high family support was the strongest predictor of good self-care among a number of psychosocial variables (Gillibrand \& Stevenson, 2006). Relationships with friends could also influence the health of emerging adults with type 1 diabetes, as pressure to be involved with peer-focused college activities may lead to excessive alcohol consumption and poor diets, each of which can have detrimental effects on diabetes-related outcomes (Ahmed, Karter, \& Liu, 2006; Franz et al., 2002). There is a lack of literature on the impact of friends on the health of emerging adults with type 1 diabetes.

The primary goal of this study is to examine whether relationships with parents and peers are linked to the health and well-being of youth with and without type 1 diabetes as they transition to emerging adulthood. We also examine whether these relations are the same for emerging adults with and without type 1 diabetes. Because relationships with parents and peers do not occur in isolation of one another, we examine the interaction between the two domains. "Cross-domain buffering" (Lepore, 1992) is a phenomenon in which the effects of unsupportive behaviors from one social domain are buffered by supportive behaviors from another social domain. Support for this idea has been found in a study of college students that showed the relation of peer influence to heavy alcohol use and alcohol problems was strongest when parent permissiveness was high and parent monitoring was low (Wood et al., 2004), as well as a study of children with chronic disease (included type 1 diabetes) that showed friend support buffered the effects of poor parent relations on psychological/ behavioral outcomes (Herzer, Umfress, Aljadeff, Ghai, \& Zakowski, 2009).

This longitudinal study examined the association of parent and friend relationships to health behaviors (e.g., alcohol intake) and psychological well-being (e.g., depressive symptoms) among youth with and without type 1 diabetes during the transition to emerging adulthood. We examined a supportive and an unsupportive/strained dimension of parent and friend relations. We predicted that supportive relationships would be associated with good health outcomes and problematic relationships would be associated with poor health outcomes. We tested cross-domain buffering by examining whether supportive relationships in one domain buffered the negative effects of problematic relationships in the other domain. We also examined whether links of relationships to health outcomes differed for those with and without diabetes. Because parents of youth with diabetes have been involved in the daily routine of diabetes self-care throughout adolescence (Vesco et al., 2010), we reasoned that parental relationships might have stronger associations with health outcomes for those with diabetes. Although parent involvement clearly declines with age (Anderson et al., 2002; Ingerski, Anderson, Dolan, \& Hood, 2010), parent involvement continues to be linked to better diabetes outcomes even among older aolescents (e.g., Helgeson, Reynolds, Siminerio, Escobar, \& Becker, 2008). We did not predict that health status would influence the association of friend relationships to health outcomes. 


\section{Methods}

\section{Participants}

Participants were 117 teens with type 1 diabetes (47.0\% male) and 122 teens without diabetes ( $46.3 \%$ male) who at baseline were in the spring semester of their senior year of high school and were 18 years old on average (diabetes: $M=18.15, S D=.41$; controls: $M=$ $18.02, S D=.49)$. Among those with diabetes, the average time since diagnosis was 11.12 years $(S D=3.10,5.75-17.74)$, the average HbA1c was $8.90 \%(S D=1.75,6.2-13.7)$, and $57.5 \%$ used an insulin pump. Table 1 lists additional demographic information at baseline for both groups.

\section{Procedure}

The appropriate Institutional Review Boards approved this study. Details on recruitment have been published elsewhere (Helgeson, Snyder, Escobar, Siminerio, \& Becker, 2007). Briefly, the original study began when participants were in the $5^{\text {th }}, 6^{\text {th }}$, and $7^{\text {th }}$ grades. The response rate was $77 \%$ for youth with type 1 diabetes, who were recruited from the local Children's Hospital. There were two sources for recruiting control subjects (66\% response rate): 61 were recruited from physicians' offices, and 70 were recruited from area malls. Participants in the present study were recruited from those who participated in the previous study. Of those from the original study, 121 (91.7\%) of the 132 participants with type 1 diabetes and 123 (93.9\%) of the 131 control participants agreed to be contacted for the current study.

Consent forms were mailed to potential participants after ascertaining interest in the study by telephone. Upon receipt of signed consent forms, teens were emailed a link to an online questionnaire both during their senior year of high school (Time 1 [T1]) and one year later (Time 2 [T2]). Paper questionnaires were sent to participants without online access or with a preference to complete a written survey. The percentage of participants who completed assessments at $\mathrm{T} 1$ and $\mathrm{T} 2$ was $97 \%$ for diabetes (117 of the 121 participants) and $99 \%$ for controls (122 of the 123). Of non-respondents, three declined participation and others were unreachable.

Demographic information on participants at $\mathrm{T} 2$ is presented in Table 1. Roughly $75 \%$ of participants were attending college and 50\% were working. Just over one-third were living at home with parents, largely those who were not in college. These demographic variables did not differ between diabetes and controls.

\section{Measures}

Participant sex, birthdate, race/ethnicity, household structure and social status (Hollingshead, 1975) were recorded from the prior study (Helgeson et al., 2007). Body mass index (BMI) was calculated from participants' self-reported current height and weight. Parent and peer relationship variables were assessed at T2; health variables were assessed at T1 and T2.

Parent support-Participants were asked to answer three questions to assess their level of closeness to their parents: (1) "How close are you to your parents?" rated on a 5-point scale 
from 1 (not at all) to 5 (very), (2) "How often do you confide in your parents?" rated on a 5point scale from 1 (never) to 5 (very often), and (3) How often are you in contact with your parents by phone, instant messaging, or email?" rated on a 7-point scale from 1 (more than once a day) to 7 (less than once a month). Participants also rated parent involvement in their lives with three scales developed by Kerr and Stattin (Kerr \& Stattin, 2000; Kerr, Stattin, \& Trost, 1999): parental monitoring (e.g., "Do your parents know what you do in your free time?"; $a=.82$ ), child disclosure of feelings (e.g., "Do you tell your parents how you really feel about things?"; $a=.90$ ), and child disclosure of activities (e.g., "Do you talk to your parents about school or work?"; $a=.73)$. Participants answered each question on a 5-point scale from 1 (never) to 5 (always). When the three single item questions and three scales were submitted to principle components analysis, all loaded on a single factor. Factor loadings ranged from .80 to .90 with the exception of the contact question which loaded .56 . Thus, that question was discarded, and the average of the two single-item questions and three scales (all standardized) was computed to represent a parent support variable. The kind of support best represented by this variable is emotional support. The internal consistency of this scale was high $(a=.89)$.

Parent control-We administered the 4-item feeling controlled by parents scale, also from Kerr and Stattin (2000). Sample items include "Do you feel as though your parents control everything in your life?" and "Do you feel that your parents demand to know everything?" This instrument was developed within the framework of the parental monitoring literature and aims to assess one way in which parents acquire information about their children-by actively controlling them. This measure reflects participants' perceptions of being controlled by parents. The internal consistency was high $(\alpha=.87)$.

Friend support—Friend support was measured by administering three support subscales from the Berndt and Keefe (1995) friendship questionnaire: intimacy $(a=.87)$, instrumental support $(a=.82)$, and emotional support $(a=.90)$. This scale has been shown to have excellent reliability and validity. The three subscales were averaged to create a global friend support index; the internal consistency was high $(\alpha=.94)$.

Friend conflict-Conflict with friends was measured with four subscales from the Test of Negative Social Exchange (Ruehlman \& Karoly, 1991): impatience $(a=.82)$, insensitivity $(\alpha=.83)$, interference $(\alpha=.70)$, and rejection $(\alpha=.86)$. The scales have high test-retest reliability, high internal consistency, and are distinct from one another as determined by factor analysis (Ruehlman \& Karoly, 1991). The average of the four subscales was taken to create a global friend conflict scale $(a=.93)$.

Smoking -We measured cigarette smoking with a question from the Monitoring the Future Study (Johnston, O'Malley, Bachman, \& Schulenberg, 2005). We asked participants how often they had smoked cigarettes in the past 12 months. We created a dichotomous variable, such that 0 indicated never smoked in the past year and 1 indicated had ever smoked in the past year. At T1, 29\% of youth reported having smoked in the past year; at T2, it was $38 \%$.

Alcohol use/binge drinking-To measure alcohol consumption and binge drinking, a series of questions from the Monitoring the Future Study was used (Johnston et al., 2005). 
Participants were asked to indicate the number of times they drank more than a few sips of alcohol during the past month and were assigned either a 1 (had consumed any alcohol) or a 0 ( had not consumed any alcohol). At T1, 32\% of youth had consumed alcohol; at T2 it was $52 \%$. Consistent with the Monitoring the Future Study, binge drinking was defined as the consumption of five or more drinks of alcohol on a single occasion in the past month for males and four or more drinks of alcohol on a single occasion for females. We created a categorical variable, such that 1 represented one or more binges and 0 represented no binges in the past month. At T1, 16\% of youth had reported a binge; at $\mathrm{T} 2$ it was $34 \%$.

Depressive symptoms-Depressive symptoms were measured by the 20-item Center for Epidemiologic Studies Depression Scale (Radloff, 1977). Participants indicated how often they experienced each symptom on a 0 (none of the time) to 3 (most of the time) scale. Items were summed to create a total score. This scale has well-established internal consistency and validity, and has been widely used with samples of emerging adults. Internal consistency in this study was high (T1: $\mathrm{a}=.89$; $\mathrm{T} 2: \mathrm{a}=.93$ ).

Perceived stress-We administered the abbreviated form (4-item) of the Perceived Stress Scale (Cohen, Kamarck, \& Mermelstein, 1983). The abbreviated measure has wellestablished reliability and validity (Cohen et al., 1983). The internal consistency was good at both assessments (T1: $\mathrm{a}=.72 ; \mathrm{T} 2: \mathrm{a}=.76)$

Disturbed eating behavior-Two subscales from the Eating Disorder Inventory (Garner, 1990) were administered: drive for thinness (excessive concern with dieting, preoccupation with weight) and bulimia (episodes of uncontrollable eating or bingeing). Three items from the drive for thinness scale were removed because they are biased by the presence of diabetes (Steel, Young, Lloyd, \& Macintyre, 1989). Their inclusion in previous research has artificially inflated the presence of eating disturbances among people with diabetes. The validity and reliability of these scales are well-established. The internal consistencies were good in the present study (drive for thinness .91 at T1 and T2; bulimia .82 at T1 and .85 at $\mathrm{T} 2)$.

Diabetes outcomes-Self-care was measured by the 14-item Self-Care Inventory (La Greca, Swales, Klemp, \& Madigan, 1988; Lewin et al., 2009), which asks respondents to indicate how well they followed their physicians' recommendations for glucose testing, insulin administration, diet, exercise, and other diabetes behaviors. This index reflects domains of self-care that have been regarded as important by the American Diabetes Association, and has been associated with glycemic control among adolescents (Delamater, Applegate, Edison, \& Nemery, 1998; Greco et al., 1990; La Greca et al., 1988). This instrument was updated by adding eight more contemporary items as described previously (Helgeson et al., 2008). The final 22 items were measured on a scale from 1 (never) to 5 (always/very often); negative items were reverse-coded, and then all were averaged to create a final self-care index. Internal consistency for this index was good (T1: $a=.85 ; \mathrm{T} 2: \mathrm{a}=$. 88). Glycemic control was measured using the participants' most recent HbA1c, which was requested from each participant's current physician. 


\section{Statistical Analysis}

First, zero-order correlations of the T2 independent variables (parent support, parent control, friend support, friend conflict) and T2 dependent variables were computed (see Table 2).

Then, dependent variables were grouped into three meaningful categories (behavioral outcomes, psychological outcomes, diabetes outcomes), and the following progression of analyses was performed for each. The four $\mathrm{T} 2$ independent variables were entered simultaneously in a regression analysis to predict each of the T2 dependent variables, controlling for the respective T1 dependent variable (Model 1; see Table 3). For dichotomous outcomes (smoking, alcohol consumption, binge drinking), logistic regression was used. Analyses were not adjusted for demographic variables because none were related to both independent and dependent variables. Analyses of diabetes outcomes were adjusted for time since diagnosis and insulin delivery method (pump vs. multiple daily injections), as these two variables were related to outcomes.

Next, we examined the interaction of health status group (diabetes or control) with each of the four independent variables (Model 2). Because there were differences in BMI and social status between diabetes and control groups, we adjusted for both of these variables on the first step of the equation. We entered the centered main effects of the four independent variables on the second step, and the interactions of each centered independent variable with health status on the final step. Because only a single interaction appeared (described below), we do not show Model 2 in Table 3.1 Thus, the data shown in Table 3 reflect both diabetes and control groups.

Finally, we tested the cross-domain buffering hypothesis by computing the interaction between parent support and friend conflict and the interaction between friend support and parent control (Model 3, significant interactions shown in Table 3). Again, we added these two interactions after relevant statistical control variables and centered independent variables.

\section{Results}

\section{Correlations Among Independent and Dependent Variables}

As shown in Table 2, the four independent variables were modestly correlated. Smoking was modestly related to alcohol use and binge drinking, whereas alcohol use and binge drinking were strongly related. Among the four psychological health outcomes, perceived stress and depressive symptoms were strongly related, the two disturbed eating behaviors were

\footnotetext{
${ }^{1}$ Because relationships are more central to the female than the male gender role (Cross \& Madson, 1997), we also examined whether parent and friend relationships showed differential relations to outcomes for males and females. We computed interactions between participant sex and each of the four independent variables. Participant sex did not interact with any of the independent variables to predict outcomes, with the exception of disturbed eating behavior. Friend support and friend conflict each interacted with participant sex to predict bulimic symptoms $(\beta=-.43, p<.01 ; \beta=.85, p<.05)$; and friend conflict interacted with participant sex to predict drive for thinness $(\beta=1.20, p<.01)$. In each case, significant findings were limited to females. Specifically, friend support predicted fewer bulimic symptoms and friend conflict predicted more bulimic symptoms and greater drive for thinness only among females. These findings not only suggest relationships with friends are an important avenue to explore in the area of eating behavior among women, but also suggest that stress might be a mediating variable as it was the conflictual rather than the supportive aspects of friend relationships that showed the more robust association.
} 
strongly related, and other correlations were moderate. As expected, self-care behavior was related to better glycemic control.

\section{Health Behavior Outcomes}

As shown in Model 1 of Table 3, parent support was associated with a reduced likelihood of smoking and parent control was related to an increased likelihood of smoking. Friend conflict predicted an increased likelihood of both drinking alcohol and binge drinking.

Only a single interaction with health status group occurred, and this appeared for smoking. The parent control by health status group interaction $(\mathrm{B}=-1.15 ; \mathrm{SE}=.45, p<.05)$ revealed that parent control was related to an increased likelihood of smoking for the control group but was unrelated to smoking status for the diabetes group.

There was evidence of cross-domain buffering on alcohol use (Model 3 in Table 3). Friend support interacted with parent control. Figure 1 shows parent control had little relation to alcohol use when friend support was high but was related to more alcohol use when friend support was low. Thus, friend support seems to buffer the negative effects of parent control.

\section{Psychological Outcomes}

As shown in Model 1 of Table 3, parent control and friend conflict were associated with an increase in depressive symptoms, whereas parent support was associated with a decrease in depressive symptoms. For perceived stress, friend conflict predicted an increase and parent support predicted a decrease over the year. Friend conflict predicted an increase in bulimic symptoms and drive for thinness. None of the four independent variables interacted with health status group to predict psychological outcomes.

There was evidence of cross-domain buffering on bulimic symptoms (Model 3 in Table 3). The interaction between parent support and friend conflict was significant. As shown in Figure 2, friend conflict had no effect under conditions of high parent support. However, high friend conflict was associated with an increase in bulimic symptoms in the presence of low parent support. Thus, parent support appeared to buffer emerging adults against the potential adverse effects of high friend conflict.

\section{Diabetes Outcomes}

Self-care-As shown in Model 1 of Table 3, parent support was related to better self-care behavior, and none of the variables was related to glycemic control. There was evidence of cross-domain buffering on both diabetes outcomes (Model 3 of Table 3). For self-care behavior, friend support interacted with parent control. As shown in Figure 3, when friend support was high, parent control had no relation to self-care behavior. However, when friend support was low, greater parent control was related to better self-care behavior. The lowest level of self-care was exhibited by emerging adults who were low in friend support and low on parent control—potentially, those youth for whom nobody was involved in their lives.

The interaction between parent support and friend conflict predicted glycemic control. Because the actual glycemic control numbers are meaningful (i.e., expectations are that emerging adults should have an Hba1c of less than 7.5), we present the unadjusted means in 
Figure 4 for easier interpretation. When parent support was low, friend conflict was associated with higher Hba1c's or poorer glycemic control. However, when parent support was high, friend conflict was associated with better glycemic control. Thus, parent support appeared to buffer emerging adults from the poorer glycemic control associated with high friend conflict. However, the interaction also suggests that emerging adults with low friend conflict and low parent support had poor glycemic control. This portion of the interaction is more difficult to explain.

\section{Discussion}

This study showed that parent relationships remained important during emerging adulthood. Supportive relationships with parents predicted better health behavior in terms of smoking (but not alcohol), higher levels of psychological well-being, and better diabetes self-careall with controls for the previous year's levels. Thus, maintaining a close relationship with parents when the environments of emerging adults are new and rapidly evolving seems to benefit both behavior and psychological well-being. Support from parents also buffered adverse effects of conflict with friends on two outcomes-bulimic symptoms and glycemic control. In both cases, friend conflict was related to worse outcomes only in the presence of low parent support. Supportive relationships with parents may provide emerging adults with the resources to better cope with conflictual relationships with friends or with resources to resist negative influences from friends. In either case, parent support appears to be a resource in and of itself that emerging adults can utilize in situations of adversity.

By contrast, there was an aspect of emerging adults' relationships with parents that was associated with poor health outcomes. Emerging adults who perceived parents as controlling reported more depressive symptoms and, in the absence of friend support, increased alcohol use. Parent control has been thought to inhibit the development of autonomy during adolescence (Petit, Laird, Dodge, Bates, \& Criss, 2001), and has been linked to both internalizing (e.g., depression) and externalizing problems among adolescents (Barber, 1996). Parental control at the age of emerging adulthood may end up leading to a phenomenon known as psychological reactance (Brehm, 1966) - a situation in which one reacts to controlling behavior by doing just the opposite of what is expected. Emerging adults who lack support from friends may be more susceptible to psychological reactance in response to parent controlling behavior. Here, friend support buffered the adverse effects of parent control on behavior. Parent control also was related to increased smoking behaviorbut only among the healthy cohort. Emerging adults with type 1 diabetes may have grown accustomed to parent control throughout their childhood and teenage years and, thus, be less bothered by it.

There is further evidence among the diabetes cohort that parental control was less harmful and possibly helpful. Emerging adults with type 1 diabetes who perceived parents as controlling enacted better self-care in the absence of friend support. These emerging adults may be more reliant on their parents. That is, their parents may be taking control and intruding into their lives in a way that ensures that they enact appropriate diabetes self-care. The net result in the short-term is that these youth do take better care of their diabetes. The net effect in the long run remains to be seen, as these youth may be less equipped to take 
care of themselves when they are truly on their own. Another study identified a condition under which parent control was beneficial. Specifically, Pettit and colleagues (2001) found that parent control was associated with more delinquent problems among youth who had low levels of delinquency as children but fewer delinquent problems among youth who had high levels of delinquency as children. The presence of type 1 diabetes does not reflect delinquency, but it may reflect a condition under which parent control can be adaptiveagain, due to the complexity in disease management.

Parent control also may be more likely to persist at this stage as just over one-third of emerging adults were living at home. We wondered if youth's living situation was related to parent control and undertook a post-hoc analysis. Living at home was not related to parent support but was related to parent control. Youth who lived at home were more likely to perceive parents as controlling, $F(1,233)=5.66, p<.05$. Parents might feel entitled to know where their children are and what they are doing when they live in the same house. However, these data were collected only the first year after high school graduation. In subsequent years as more young adults leave home, it may be more difficult for parents to maintain control, and emerging adults' psychosocial development may be impeded if they are overly reliant on parents.

Relationships with friends also were linked to health outcomes, but it was the conflictual rather than the supportive aspects of these relationships that had the most consistent links. Consistent with previous research (Palladino \& Helgeson, 2012), friend conflict was a more robust predictor of outcomes than friend support. Support is an expected norm of friendship. Thus, its presence may be less salient and less influential than conflict on behavior and wellbeing. Conflict with friends predicted increased alcohol use and increased binge drinking. Conflict with friends may lead to self-medication or may inspire emerging adults to drink alcohol to fit in with friends. To understand this finding more clearly, one needs to know the nature of conflict with friends. Conflict with friends also predicted increases in all psychological distress outcomes—depressive symptoms, perceived stress, bulimic symptoms, and drive for thinness.

The hypothesis that supportive relationships with parents could buffer the adverse effects of conflict with friends, or that supportive friend relationships could buffer the adverse effects of problematic relationships with parents received some support. We found cross-domain buffering on one of the health behavior outcomes, one of the psychological outcomes, and both of the diabetes outcomes. Taken collectively, these findings suggest that relationships with parents and peers are not only critical during the early years of emerging adulthood but that they have a synergistic effect. Although parents may feel that this transition is an appropriate time to increase emotional separation from their children to allow them to grow into adults, these findings suggest that continued closeness may be beneficial, particularly for well-being and diabetes health. However, it is also the case that parents need to support emerging adults' autonomy rather than dependence, as parent controlling behavior was associated with adverse outcomes. It remains for future research to understand the mechanisms that underlie how support from one system can offset the negative effects of problems with the other social system. 
It was anticipated that there would be a stronger association of parent support to outcomes for those with than without diabetes during emerging adulthood. This was not the case. Parent support did not interact with health status to predict health behaviors or psychological well-being. The only aspect of parent relationships that did have differential relations to outcomes was parent control-and this occurred in only one instance. Parental influence may have been similar for youth with and without diabetes because it was only the first year of separation for most of our sample. Differential effects may emerge in later years if parents of healthy youth decrease monitoring at a higher rate than parents of youth with diabetes. However, parent support was critical to those with diabetes because it was related to both diabetes outcomes. Parent support was associated with better diabetes self-care and buffered the negative relation of friend conflict to poor glycemic control.

The continued importance of parent relationships for psychological and diabetes-related health has important implications for clinical care. Although many youth transfer to adult healthcare at some point during emerging adulthood, it is important for clinicians to be aware of the benefit of high quality parent-child relationships, and to consider the risk that may exist when relationships with parents are strained, lacking, or controlling. Healthcare professionals should also be aware of emerging adults' peer relationships and the implications that problems with friends may have for health. Careful screening for problems with family and peer relationships at this age should be included in any medical or psychological screening process to bring attention to any potential detriment to health and well-being.

Before concluding, it is important to note a few study limitations. Respondents were primarily middle class and Caucasian, limiting generalizability to minority and lower social status populations who may have less opportunity to engage in the same level of exploration during emerging adulthood (Arnett, 2004), and whose relationships with parents and friends may be culturally distinct. Although we measured a supportive and an unsupportive aspect of both parent and friend relationships, the measures were not the same. For example, our measure of parent support focused on emotional support, whereas the measure of friend support represented both emotional and instrumental aspects of support. Although the study is longitudinal and we controlled for baseline levels of the dependent variables to examine changes over time, we cannot draw causal conclusions from these data. The social environment may affect health behaviors and well-being, health behaviors and well-being may influence the social environment, or other variables (such as living situation and vocation) could influence both sets of variables. In addition, only the first year of emerging adulthood was examined; relations found during this first year may transform as youth age.

It is important to follow this sample as they progress toward adulthood to study any changing associations of parent and friend relationships with behavior and health. However, intervening during the first year of emerging adulthood could be an important step for avoiding the establishment of undesirable behaviors that may persist into adulthood. Managing self-care and maintaining tight glycemic control is crucial for mitigating diabetesrelated complications that may become more clinically significant as youth age (Alleyn et al., 2010). With rising diagnosis of type 1 diabetes expected worldwide for decades to come (Peters \& Laffel, 2011) and the important fluctuations in the social environment that occur 
between adolescence and adulthood, understanding how the social environment may influence diabetes health and well-being during emerging adulthood will continue to be an important area of study.

These findings suggest that relationships with both parents and friends remain important predictors of health behaviors and psychological well-being during emerging adulthood. Further, the quality of parent and friend relationships are related to type 1 diabetes outcomes, extending the known importance of these social relationships during adolescence into emerging adulthood.

\section{Acknowledgements}

This work was supported by National Institutes of Health grant R01 DK60586. We appreciate the support of the project manager, Pamela Snyder, and the research assistants, Abigail Vaughn and Jamie Vance, for their assistance on this project.

\section{References}

Ahmed AT, Karter AJ, Liu J. Alcohol consumption is inversely associated with adherence to diabetes self-care behaviours. Diabetic Medicine. 2006; 23:795-802. [PubMed: 16842486]

Alleyn CR, Volkening LK, Wolfson J, Rodriguez-Ventura A, Wood JR, Laffel LM. Occurrence of microalbuminuria in young people with Type 1 diabetes: importance of age and diabetes duration. Diabetic Medicine. 2010; 27:532-537. [PubMed: 20536948]

Allison, GC. The influences of gender, parents, and friends on the alcohol-related problems of college freshmen. Tulane University; New Orleans, LA: 2003.

American Psychiatric Association. Diagnostic and statistical manual of mental disorders. Fourth ed.. American Psychiatric Association; Washington, DC: 2000.

Anderson B, Vangsness L, Connell A, Butler D, Goebel-Fabbri A, Laffel L. Family conflict, adherence, and glycaemic control in youth with short duration type 1 diabetes. Diabetic Medicine. 2002; 19:635-642. [PubMed: 12147143]

Arnett JJ. Emerging adulthood: A theory of development from the late teens through the twenties. American Psychologist. 2000; 55:469-480. [PubMed: 10842426]

Arnett, JJ. Emerging adulthood: The winding road from the late teens through the twenties. Oxford University Press; New York: 2004.

Arnett, JJ. Socialization in emerging adulthood: From the family to the wider world, from socialization to self-socialization. In: Gruesec, JE.; Hastings, PD., editors. Handbook of socialization: Theory and research. Guilford Press; New York: 2007. p. 208-231.

Barber B. Parental psychological control: Revisiting a neglected construct. Child Development. 1996; 67:3296-3319. [PubMed: 9071782]

Berndt TJ, Keefe K. Friends' Influence on Adolescents' Adjustment to School. Child Development. 1995; 66:1312-1329. [PubMed: 7555218]

Borsari B, Carey KB. Peer influences on college drinking: A review of the research. Journal of Substance Abuse. 2001; 13:391-424. [PubMed: 11775073]

Brehm, JW. A theory of psychological reactance. Academic Press; 1966.

Brown, RT.; Boeving, A.; LaRosa, A.; Carpenter, LA. Health and Chronic Illness. In: Wolfe, DA.; Mash, EJ., editors. Behavioral and emotional disorders in adolescents: Nature, assessment, and treatment. Guilford Publications; New York: 2006. p. 505-531.

Cohen S, Kamarck T, Mermelstein R. A global measure of perceived stress. Journal of Health and Social Behavior. 1983; 24:385-396. [PubMed: 6668417]

Cross SE, Madson L. Models of the self: Self-construal and gender. Psychological Bulletin. 1997; 122:5-37. [PubMed: 9204777] 
Delamater A, Applegate B, Edison M, Nemery R. Increased risks for poor metabolic control in minority youths with type 1 diabetes. Diabetes. 1998; 47:A326.

Franz MJ, Bantle JP, Beebe CA, Brunzell JD, Chiasson J-L, Garg A, et al. Evidence-Based Nutrition Principles and Recommendations for the Treatment and Prevention of Diabetes and Related Complications. Diabetes Care. 2002; 25:148-198. [PubMed: 11772915]

Frech, AM. Healthy behavior trajectories between adolescence and emerging adulthood. Ohio State University; Columbus, OH: 2009.

Garner, DM. Eating disorder inventory-2. Psychological Assessment Resources, Inc; Odessa, FL: 1990.

Gillibrand R, Stevenson J. The extended health belief model applied to the experience of diabetes in young people. British Journal of Health Psychology. 2006; 11:155-169. [PubMed: 16480561]

Greco P, LaGreca A, Ireland S, Wick P, Freeman C, Agramonte R, et al. Assessing adherence in IDDM: A comparison of two methods. Diabetes. 1990; 40:A165.

Haemmerlie FM, Steen SC, Benedicto JA. Undergraduates' conflictual independence, adjustment, and alcohol use: The importance of the mother-student relationship. Journal of Clinical Psychology. 1994; 50:644-650. [PubMed: 7983216]

Helgeson VS, Reynolds KA, Siminerio L, Escobar O, Becker D. Parent and adolescent distribution of responsibility for diabetes self-care: Links to health outcomes. Journal of Pediatric Psychology. 2008; 33:497-508. [PubMed: 17848390]

Helgeson VS, Snyder PR, Escobar O, Siminerio L, Becker D. Comparison of adolescents with and without diabetes on indices of psychosocial functioning for three years. Journal of Pediatric Psychology. 2007; 32:794-806. [PubMed: 17426042]

Herzer M, Umfress K, Aljadeff G, Ghai K, Zakowski SG. Interactions with parents and friends among chronically ill children: Examining social networks. Journal of Developmental \& Behavioral Pediatrics. 2009; 30:499-508. [PubMed: 19861900]

Hollingshead, AB. Four factor index of social status. Yale University; New Haven, CT: 1975.

Holmbeck GN. A developmental perspective on adolescent health and illness: An introduction to the special issues. Journal of Pediatric Psychology. 2002; 27:409-416. [PubMed: 12058005]

Holmbeck, GN.; Friedman, D.; Abad, M.; Jandasek, B. Development and Psychopathology in Adolescence. In: Wolfe, DA.; Mash, EJ., editors. Behavioral and emotional disorders in adolescents: Nature, assessment, and treatment. Guilford Publications; New York: 2006. p. 21-55.

Ingerski LM, Anderson BJ, Dolan LM, Hood KK. Blood gluclose monitoring and glycemic control in adolescence: Contribution of diabetes-specific responsibility and family conflict. Journal of Adolescent Health. 2010; 47:191-197. [PubMed: 20638012]

Johnston, LD.; O'Malley, PM.; Bachman, JG.; Shulenberg, JE. Monitoring the future national survey results on drug use, 1975-2004: Volume II, College students and adults ages 19-45. National Institute on Drug Abuse; Bethesda: 2005.

Kerr M, Stattin H. What parents know, how they know it, and several forms of adolescent adjustment: Further support for a reinterpretation of monitoring. Developmental Psychology. 2000; 36:366380. [PubMed: 10830980]

Kerr M, Stattin H, Trost K. To know you is to trust you: parents' trust is rooted in child disclosure of information. Journal of Adolescence. 1999; 22:737-752. [PubMed: 10579887]

Kins E, Beyers W, Soenens B, Vansteenkiste M. Patterns of home leaving and subjective well-being in emerging adulthood: The role of motivational processes and parental autonomy support. Developmental Psychology. 2009; 45:1416-1429. [PubMed: 19702402]

Kuther TL, Higgins-D'Alessandro A. Attitudinal and normative predictors of alcohol use by older adolescents and young adults. Journal of Drug Education. 2003; 33:71-90. [PubMed: 12773026]

La Greca, AM.; Swales, T.; Klemp, S.; Madigan, S. Self care behaviors among adolescents with diabetes. Paper presented at the Ninth Annual Sessions of the Society of Behavioral Medicine. Paper presented at the Society of Behavioral Medicine; Baltimore, MD. 1988.

Lepore SJ. Social conflict, social support, and psychological distress: Evidence of cross-domain buffering effects. Journal of Personality and Social Psychology. 1992; 63:857-867. [PubMed: 1447698] 
Lewin AB, Heidgerken AD, Geffken GR, Williams LB, Storch EA, Gelfand KM, et al. The relation between family factors and metabolic control: the role of diabetes adherence. Journal of Pediatric Psychology. 2006; 31:174-183. [PubMed: 16467317]

Lewin AB, LaGreca AM, Geffken GR, Williams LB, Duke DC, Storch EA, et al. Validity and reliability of an adolescent and parent rating scale of type 1 diabetes adherence behaviors: the SelfCare Inventory (SCI). Journal of Pediatric Psychology. 2009; 34:999-1007. [PubMed: 19423660]

Palladino DK, Helgeson VS. Friends or foes? A review of peer influence on self-care and glycemic control in adolescents with type 1 diabetes. Journal of Pediatric Psychology. 2012; 37:591-603. [PubMed: 22460759]

Peters A, Laffel L. Diabetes care for emerging adults: Recommendations for transition from pediatric to adult diabetes care systems. Diabetes Care. 2011; 34:2477-2485. [PubMed: 22025785]

Pettit GS, Laird RD, Dodge KA, Bates JE, Criss MM. Antecedents and behavior-problem outcomes of parental monitoring and psychological control in early adolescence. Child Development. 2001; 72:583-598. [PubMed: 11333086]

Radloff LS. The CES-D Scale: A Self-Report Depression Scale for Research in the General Population. Applied Psychological Measurement. 1977; 1:385-401.

Ramchandani N, Cantey-Kiser JM, Alter CA, Brink SJ, Yeager SD, Tamborlane WV, et al. Selfreported factors that affect glycemic control in college students with type 1 diabetes. The Diabetes Educator. 2000; 26:656-666. [PubMed: 11140074]

Ruehlman LS, Karoly P. With a little flak from my friends: Development and preliminary validation of the Test of Negative Social Exchange (TENSE). Psychological Assessment: A Journal of Consulting and Clinical Psychology. 1991; 3:97-104.

Sessa FM. The influence of perceived parenting on substance use during the transition to college: A comparison of male residential and commuter students. Journal of College Student Development. 2005; 46:62-74.

Steel JM, Young RJ, Lloyd GG, Macintyre CC. Abnormal eating attitudes in young insulin-dependent diabetics. The British Journal of Psychiatry. 1989; 155:515-521. [PubMed: 2611574]

Steinberg L, Monahan KC. Age differences in resistance to peer influence. Developmental Psychology. 2007; 43:1531-1543. [PubMed: 18020830]

Vesco AT, Anderson B, Laffel L, Dolan LM, Ingerski LM, Hood KK. Responsibility sharing between adolescents with type 1 diabetes and their caregivers: Importance of adolescent perceptions on diabetes management and control. Journal of Pediatric Psychology. 2010; 35:1168-1177. [PubMed: 20444852]

Wood MD, Read JP, Mitchell RE, Brand NH. Do parents still matter? Parent and peer influences on alcohol involvement among recent high school graduates. Psychology of Addictive Behaviors. 2004; 18:19-30. [PubMed: 15008682] 




Figure 1.

The relation of parent control to the percentage of youth who used alcohol for those with low and high friend support. 


$$
2.50
$$

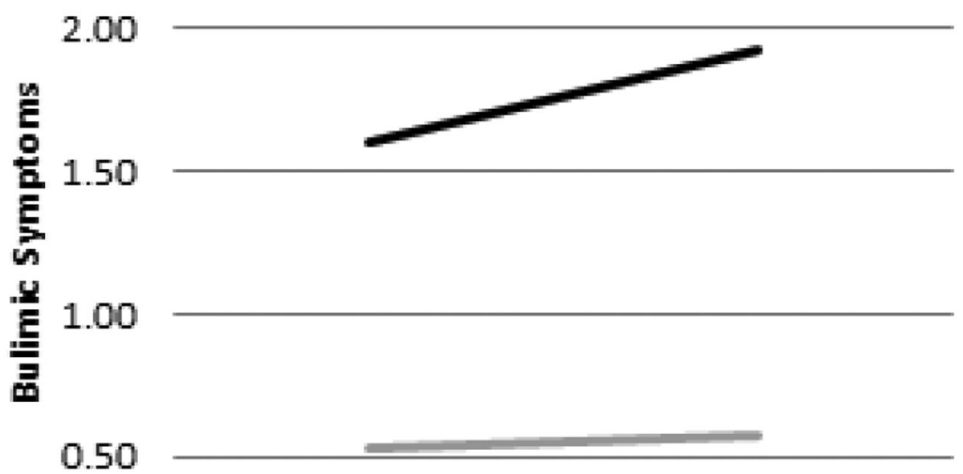

0.00



Friend Conflict

-Parent Support Low —Parent Support High

Figure 2.

The relation of friend conflict to bulimic symptoms for those who have low and high parent support. 


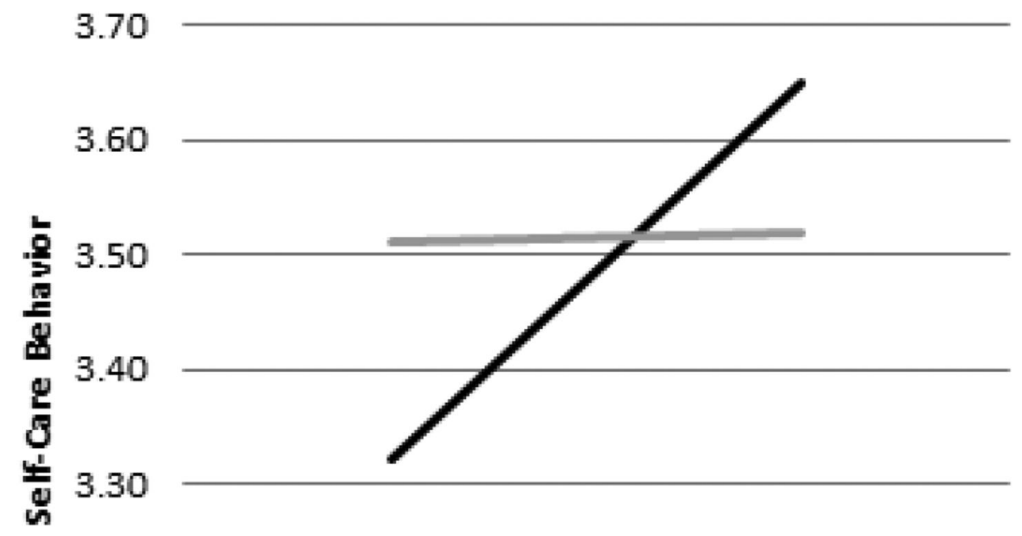

$$
3.20
$$

\subsection{0}

Low

Parent Control



Figure 3.

The relation of parent control to self-care behavior among those who have low and high friend support. 


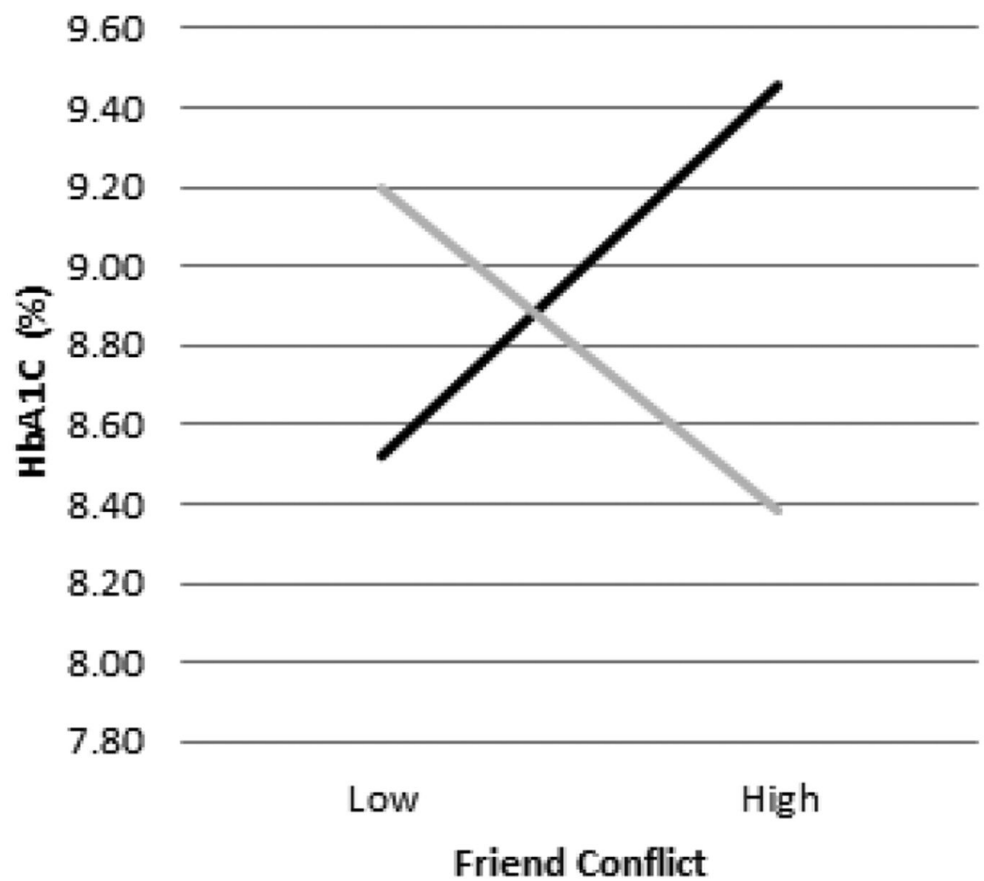

- Parent Support Low $=$ Parent Support High

Figure 4.

The relation of friend conflict to glycemic control (hbA1c) among those who have low and high parent support. 
Table 1

Participant Demographics at Baseline (T1) and Follow-up (T2)

\begin{tabular}{lcc}
\hline & Diabetes $(\boldsymbol{n}=\mathbf{1 1 7})$ & Controls $(\boldsymbol{n}=\mathbf{1 2 2})$ \\
\hline BASELINE (T1) & & \\
Body mass index ${ }^{*}$ & $25.68(4.03)$ & $24.12(4.72)$ \\
Social status (Hollingshead) ${ }^{*}$ & $42.61(11.08)$ & $46.58(13.70)$ \\
Household status: \% Lives w/mother and father & $66.7 \%$ & $69.4 \%$ \\
Race: \% White & $92.3 \%$ & $93.4 \%$ \\
Ethnicity: \% Hispanic & $1.7 \%$ & $3.3 \%$ \\
FOLLOWUP (T2) & & \\
Full-time college (\%) & $75.2 \%$ & $74.6 \%$ \\
Working (\%) & $52.1 \%$ & $52.5 \%$ \\
Living at home (\%) & $37.0 \%$ & $36.0 \%$ \\
\hline
\end{tabular}

Note: Birthdate, sex, social status, household structure, race, and ethnicity were collected from the original study when participants were average age 12 (Helgeson et al., 2007); all remaining data were collected at T1.

* health status difference at $p<.05$; 


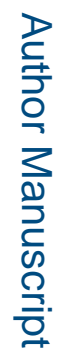



Health Psychol. Author manuscript; available in PMC 2016 November 03. 


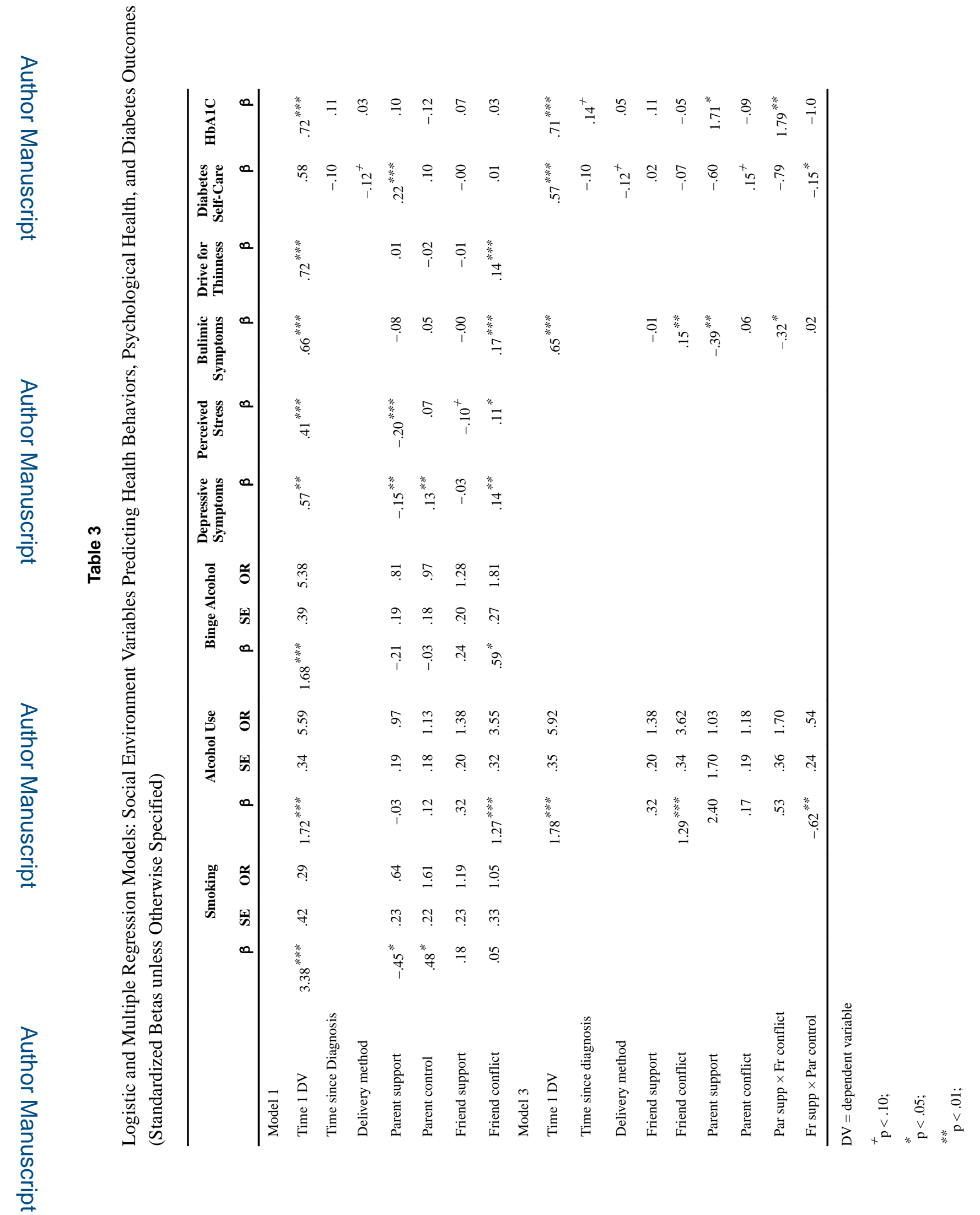

Health Psychol. Author manuscript; available in PMC 2016 November 03. 

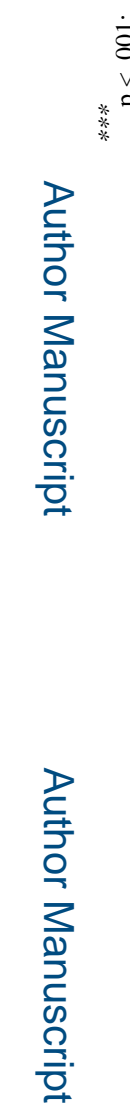



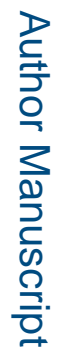

Health Psychol. Author manuscript; available in PMC 2016 November 03. 